PROCEEDINGS OF THE

AMERICAN MATHEMATICAL SOCIETY

Volume 128, Number 3, Pages 713-715

S 0002-9939(99)05071-6

Article electronically published on March 3, 1999

\title{
QUASI-ISOMORPHISMS OF KOSZUL COMPLEXES
}

\author{
JOSÉ J. M. SOTO
}

(Communicated by Wolmer V. Vasconcelos)

\begin{abstract}
Let $f: A \rightarrow B$ be a surjective homomorphism of noetherian local commutative rings that induces an isomorphism between the first Koszul homology modules and an epimorphism between the second Koszul homology modules. Then $f$ induces isomorphisms between Koszul homology modules in all dimensions.
\end{abstract}

Let $(A, \mathfrak{m}, k)$ be a local noetherian (commutative with unit) ring, $I$ an ideal of $A, B=A / I$, and $\mathfrak{n}$ the maximal ideal of $B$. Let $\left\{x_{1}, \ldots, x_{n}\right\}$ be a minimal set of generators of the ideal $\mathfrak{m}$ of $A$, and $y_{1}, \ldots, y_{n}$ the images of these elements in $B$. Let $\left\{v_{1}, \ldots, v_{r}\right\}$ be a minimal set of generators of the ideal $\mathfrak{n}$ of $B$. Fix for each $1 \leq j \leq n$ elements $b_{j \alpha} \in B$ such that $y_{j}=\sum_{\alpha=1}^{r} b_{j \alpha} v_{\alpha}$. Let $E=A\left\langle X_{1}, \ldots, X_{n} ; d X_{i}=\right.$ $\left.x_{i}\right\rangle$ be the Koszul complex associated to the elements $x_{1}, \ldots, x_{n}$ of $A$, and $E^{\prime}=$ $B\left\langle V_{1}, \ldots, V_{r} ; d V_{i}=v_{i}\right\rangle$ be the Koszul complex associated to the elements $v_{1}, \ldots, v_{r}$ of $B$. By a little abuse of language we denote $H_{*}(A)=H_{*}(E), H_{*}(B)=H_{*}\left(E^{\prime}\right)$ (they do not depend, up to isomorphism, of the minimal set of generators of the maximal ideal). Let $f: E \rightarrow E^{\prime}$ be the homomorphism of complexes extending the projection map $A \rightarrow B$ by sending $X_{i}$ to $\sum_{\alpha=1}^{r} b_{j \alpha} V_{\alpha}$.

L. L. Avramov and E. S. Golod [4, Proposition 1] show that if the ideal $I$ is generated by a regular sequence which is part of a minimal system of generators of the ideal $\mathfrak{m}$, then $H_{*}(f): H_{*}(A) \rightarrow H_{*}(B)$ is an isomorphism. In [8] (see [9, (2.3.6)] S. S. Strogalov shows that the converse also holds. The following result shows that it is enough to consider the first two homology modules:

Proposition 1. If $H_{1}(f)$ is an isomorphism and $H_{2}(f)$ is surjective, then the ideal $I$ is generated by a regular sequence which is part of a minimal system of generators of the ideal $\mathfrak{m}$.

Proof. Let $0 \rightarrow U \rightarrow F \stackrel{p}{\rightarrow} \mathfrak{m} \rightarrow 0$ be an exact sequence of $A$-modules with $F$ free with basis $\left\{z_{1}, \ldots, z_{n}\right\}$ and $p\left(z_{i}\right)=x_{i}, 1 \leq i \leq n$, and let $0 \rightarrow U^{\prime} \rightarrow F^{\prime} \stackrel{p^{\prime}}{\longrightarrow} \mathfrak{n} \rightarrow 0$ be an exact sequence of $B$-modules with $F^{\prime}$ free with basis $\left\{z_{1}^{\prime}, \ldots, z_{r}^{\prime}\right\}$ and $p^{\prime}\left(z_{i}^{\prime}\right)=v_{i}$, $1 \leq i \leq r$. Let $g: F \wedge F \rightarrow F, g(a \wedge b)=p(a) b-p(b) a$, and $V=\operatorname{Im}(g)$. Define similarly $g^{\prime}: F^{\prime} \wedge F^{\prime} \rightarrow F^{\prime}$ and $V^{\prime}=\operatorname{Im}\left(g^{\prime}\right)$. We have isomorphisms $U / V=H_{1}(A)$, $U^{\prime} / V^{\prime}=H_{1}(B)$.

Received by the editors February 10, 1997 and, in revised form, May 5, 1998.

1991 Mathematics Subject Classification. Primary 13H10, 13 D03.

Key words and phrases. Koszul homology, regular sequence, complete intersection.

(c)1999 American Mathematical Society 
Consider now the André-Quillen homology modules [1], [6]. We have a commutative diagram of $k$-vector spaces with exact rows [1, 15.12]

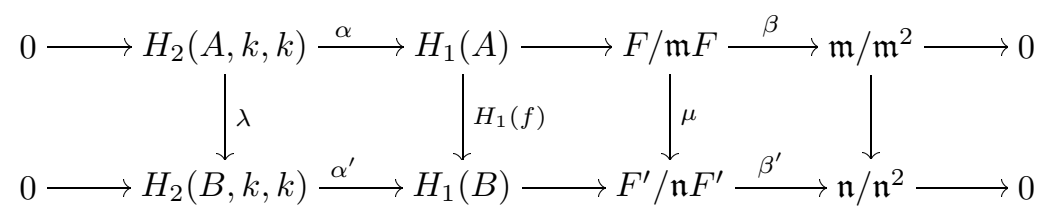

where $\mu$ is the map such that $\mu\left(z_{j}+\mathfrak{m} F\right)=\sum_{\alpha=1}^{r} b_{j \alpha}\left(z_{\alpha}^{\prime}+\mathfrak{n} F^{\prime}\right), 1 \leq j \leq n$, and $\lambda$ is the canonical map.

The maps $\beta$ and $\beta^{\prime}$ are isomorphisms and so $\alpha$ and $\alpha^{\prime}$ are also isomorphisms. Since $H_{1}(f)$ is an isomorphism by hypothesis, we see that $\lambda$ is an isomorphism.

Consider the commutative diagram of exact rows $[6,10.4]$ :

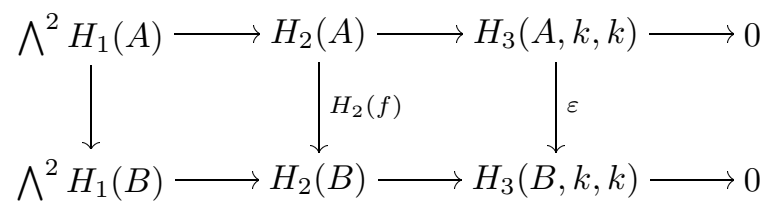

Since $H_{2}(f)$ is surjective we have that $\varepsilon$ is surjective and so from the JacobiZariski exact sequence $[1,5.1]$ associated to $A \rightarrow B \rightarrow k$

$$
H_{3}(A, k, k) \stackrel{\varepsilon}{\rightarrow} H_{3}(B, k, k) \rightarrow H_{2}(A, B, k) \rightarrow H_{2}(A, k, k) \stackrel{\sim}{\longrightarrow} H_{2}(B, k, k)
$$

we have $H_{2}(A, B, k)=0$, i.e., the ideal $I$ is generated by a regular sequence [1, 6.25].

Finally, the same Jacobi-Zariski exact sequence

$$
H_{2}(A, k, k) \stackrel{\sim}{\longrightarrow} H_{2}(B, k, k) \rightarrow H_{1}(A, B, k)=I / \mathfrak{m} I \rightarrow H_{1}(A, k, k)=\mathfrak{m} / \mathfrak{m}^{2}
$$

shows that $I / \mathfrak{m} I \rightarrow \mathfrak{m} / \mathfrak{m}^{2}$ is injective.

Remarks. i) We can see in the proof that in order to assure that $I$ is generated by a regular sequence, it is enough to assume that $H_{1}(f)$ is injective and $H_{2}(f)$ surjective.

ii) If the ideal $I$ is of finite projective dimension and $H_{2}(f)$ is surjective, then $I$ is generated by a regular sequence. The proof is the same, having in mind that by the assumption on the projective dimension of $I$, a result of L. L. Avramov [3] (see [7, Lemma 1]) gives us that $H_{2}(A, k, k) \rightarrow H_{2}(B, k, k)$ is injective.

Corollary 2. If $H_{1}(f)$ is an isomorphism and $B$ is a complete intersection ring, then $A$ is complete intersection.

Proof. It suffices to show that $H_{2}(f)$ is surjective. In the commutative diagram

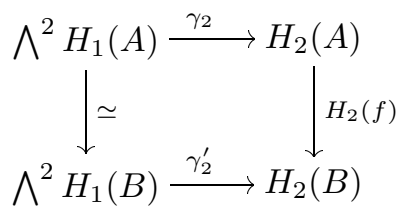

we know that $\gamma_{2}^{\prime}$ is an isomorphism [10, Theorem 6], and so $H_{2}(f)$ is surjective. 
Remark. If $A$ contains a field, $B$ is complete intersection and $H_{1}(f)$ is injective, then $A$ is complete intersection: from the diagram

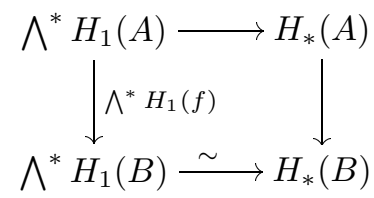

we deduce that $\Lambda^{*} H_{1}(A) \rightarrow H_{*}(A)$ is injective. Then it follows from a result of W. Bruns [5], based on deep results on commutative algebra, that $A$ is complete intersection.

We end this paper with a slight generalization of a result of E. F. Assmus. Let $(A, \mathfrak{m}, k)$ be a local noetherian ring. We know that $A$ is complete intersection if and only if the canonical homomorphism $\bigwedge^{*} H_{1}(A) \rightarrow H_{*}(A)$ is an isomorphism, i.e., $H_{*}(A)$ is a free graded (anticommutative) $k$-algebra generated by the degree 1 elements. The "only if" part is [10, Theorem 6], and the "if" part is [2, Theorem 2.7] (see also [2] and [5] for related characterizations). This result by Assmus can be stated in a slightly stronger form:

Proposition 3. If $H_{*}(A)$ is a free graded $k$-algebra, then $A$ is complete intersection.

Proof. Let $H_{*}(A)=\mathbf{S}^{*} M$, where $M$ is a graded $k$-module and $\mathbf{S}^{*}$ denotes the graded symmetric $k$-algebra functor (i.e., symmetric $k$-algebra $S$ on the even degree part, and exterior $k$-algebra $\wedge$ on the odd degree part). By [2, Theorem 2.7] it suffices to show that $M_{2}=0$. We have $S^{n} M_{2} \subset H_{2 n}(A)$ for all $n \geq 0$, and so if $M_{2} \neq 0$ we would have $H_{2 n}(A) \neq 0$ for all $n$.

\section{REFERENCES}

1. M. André, Homologie des Algèbres Commutatives, Springer, Berlin (1974). MR 50:4707

2. E. F. Assmus, On the homology of local rings, Illinois J. Math. 3 (1959), 187-199. MR 21:2670

3. L. L. Avramov, Descente des déviations par homomorphismes locaux et génération des ideaux de dimension projective finie, C. R. Acad. Sci. Paris 295 (1982), 665-668. MR 85a:14007

4. L. L. Avramov and E. S. Golod, Homology algebra of the Koszul complex of a local Gorenstein ring, Math. Notes Acad. Sci. USSR 9 (1971), 30-32. MR 43:4883

5. W. Bruns, On the Koszul algebra of a local ring, Illinois J. Math. 37 (1993), 278-283. MR 94c: 13012

6. D. Quillen, On the (co-)homology of commutative rings, Proc. Symp. Pure Math. 17 (1970), 65-87. MR 41:1722

7. A. G. Rodicio, On a result of Avramov, Manuscripta Math. 62 (1988), 181-185. MR 89k:13014

8. S. S. Strogalov, Quasiisomorphisms of a Koszul complex. (Russian) Uspehi Mat. Nauk 28 (1973), no. 6 (174), 196. MR 52:13822

9. S. S. Strogalov, On Poincaré series, Math. USSR Izvestija 12 (1978), 391-425.

10. J. Tate, Homology of noetherian rings and local rings, Illinois J. Math. 1 (1957), 14-27. MR 19:119b

Departamento de Álgebra, Facultad de Matemáticas, Universidad de Santiago de Compostela, E-15771 Santiago de Compostela, Spain 